\title{
COMUNICACIÓN
}

\section{DIAGNÓSTICO Y TRATAMIENTO DE VESÍCULAS UVEALES EN CANINOS}

\author{
Cristina Meneses B. ${ }^{1}$ y Mario Serna A. ${ }^{2}$
}

\begin{abstract}
The surgical treatment of uveal cysts in three dogs is reported. This pathology is not very common in canines and is rarely described in the literature. Uveal cysts are filled with aqueous humor and surrounded by a pigmentary epithelium, which is sometimes colorless. The origin of these formations is not well known, although they can appear after an inflammatory process (ciliary uveitis). They occur spontaneously in older animals or in cases of iris degeneration. These iridal or ciliary cysts can be uni or bilateral, single or multiple. They originate in the posterior chamber of the ciliary body and may be evident only with mydriasis or at gonioscopy (or both). Cysts of the iris are often observed as free-floating structures within the anterior chamber, having passed through the pupil or, less commonly, free-floating in the posterior chamber. The symptoms become more acute as the number of cysts increases. If they obstruct the drain angle, intraoccular pressure gradually increases (secondary glaucoma). Three dogs aged 8, 9 and II years were treated. All suffered from decreased visual acuity and their owners reported strange formations in the eyes. Intraocular pressures were 26,27 and $30 \mathrm{~mm} . \mathrm{Hg}$. The uveal cysts were surgically removed via limbal incision with $N^{\circ} 18$ needle aspiration and all three patients had satisfactory results.
\end{abstract}

Key words: Uveal cysts, glaucoma, intraocular pressure.

Palabras clave: Vesículas uveales, glaucoma, presión intraocular.

La patología de quistes uveales (Figura 1) está poco difundida en la bibliografia recopilada y en general son considerados benignos aunque se han detectado cambios en la presión intraocular debido a su presencia en la cámara anterior causando modificaciones a nivel del ángulo de drenaje.

Se atendieron tres caninos de 8,9 y 11 años de edad, dos ovejeros alemán y un mestizo (Figuras 2 y 3). Los animales presentaban visión disminuída, secreciones oculares y ha-

\footnotetext{
'Encargada del Area Especializada de Ofialmologia Facultad de l'eterinaria - Montevideo - (Jruguay.

"Director Técnico de léterinaria "Cirito de Gloria"y "Su-C'an" Montevideo - ('rugual. E.mail. crismarla'cadinet.com.uy:
}

ber detectado los propietarios "formaciones extrañas en los ojos". Al examen oftalmológico se observaron vesículas quisticas en cámara anterior en ambos ojos.

En los tres casos se realizó el control de la presión intraocular con Tonopen, obteniendo valores de 26,27 y $30 \mathrm{~mm}$. Hg. y se detectaron numerosos quistes que se encontraban afectando la visión de los pacientes, causando dolor por el aumento de la presión intraocular, por lo tanto se resolvió realizar la cirugía en los tres casos.

Mediante el uso de la oftalmoscopía directa e indirecta se inspeccionaron cámara anterior y posterior con el propósito de identificar las masas intraoculares diagnosticadas. 
Con cl Tonopen ${ }^{\text {Mr }}$ se realizó un registro de las presiones intraoculares pre y post operatorias.

La ultrasonografia fue utilizada para realizar el diagnóstico diferencial entre masas tumorales ! quistes uveales (Figuras + y 5). Los quistes son vesiculas huecas que se pueden encontrar libres en el humor acuoso, en cambio las neoplasias son macisas y compactas encontrándose siempre adheridas a la base de la úrea (Brooks, 1999; Gelatt, er al. 1979; Gelatt 1999: Slatter, 1992 ! Stades. 1999).

Mediante la transiluminación con lámpara de hendidura se puede realizar el diagnóstico diferencial con las neoplasias que no se pueden transiluminar, apareciendo como masas sólidas (Brooks, 1999).

Se utilizó como midriático Tropicannide al $1 \%$ con el propósito de visualizar la cámara posterior y los quistes.

Fue utilizada Brinzolamida al 1\% para disminuir la formación del humor acuoso mediante la inhibición de la enzima anhidrasa carbónica y Manitol al $20 \%$ a razón de $8 \mathrm{ml}$ kg I/V para controlar el aumento de la presión intraocular.

A pesar de la terapia médica la presión intraocular continuó aumentando a 26. 27 y $30 \mathrm{~mm}$. Hg., por lo que se decidió realizar la extracción de los quistes mediante cirugía. Se utilizó Tropicamide tópica al 1\% logrando midriasis y vasoconstricción durante la intervención.

Durante el abordaje a cámara anterior para realizar la extracción de los quistes se seleccionó como viscoelástico el hialuronato de sodio y condroitinsulfato por su excelente elasticidad para proteger la integridad de la córnea (Figura 6 ).
Con el paciente bajo anestesia general inhalatoria (Lsoflorano), se realizó una cantotomía y luego un abordaje a cimara anterior a traves de una incision a nis del del limbo escleral. en la región dorsal del globo ocular (a las $10.00 \mathrm{~h}$ ).

La incisión constú de pocos milimetros de forma tal que permitió la penctración de una canula (calibre 18) (Figura 7).

Al entrar en cimara anterior hay salida de humor acuoso. la tonicidad se repuso introduciendo material viscoeláitico. Para maritener la midriasis durante la cirugia e

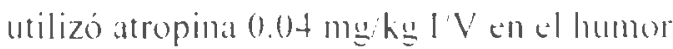
acuoso. Se aspiraron las vesiculas con lat cánula, realizando un racio o tratándolas do romper. Luego de extraidas se realizo la sutura con material absorbible $8-0$ a nicel del limbo escleral suturándose la conjuntiva. realizándo el cierre de la cantotomía (Figura 8 ).

En los tres casos se utilizé collat isabelino hasta la total cicatrizacion.

A las 24 h se controló la presion. 1 arificando una disminución de la misma ( 18 16 y $20 \mathrm{~mm} \mathrm{Hy}$. respectivamente).

El proceso inflamatorio postequirúrgico fue reducido, no lubbo caida de la presión que pudiera indicar una useitis postoperatoria significatira. La cicatrización evolucionó farorablemente, presentandose edema de cornea nínimo que desaparcició al cabo de 7 dias aproximadamente. A los 7 y a los 15 dias al controlar la presión se manturo estable (Figura 9)

Se concluse que los tres pacientes intervenidos quirúrgicamente mantusieron su presión dentro de los valones nomales. La cicatriz fue reducida! el edema de comea resultante fue mínimo. 


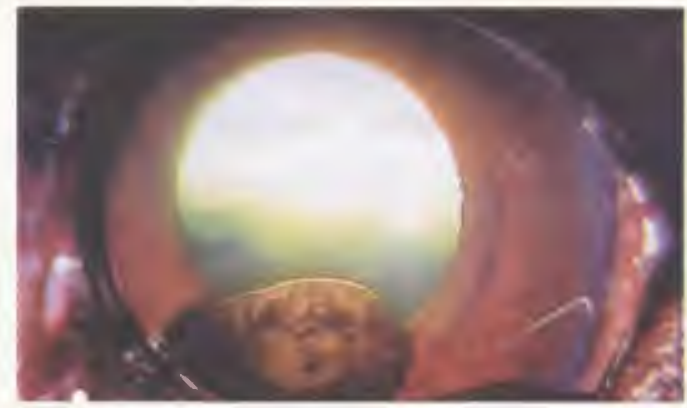

Fig 1. Quiste uveal en cámara anterior del ojo

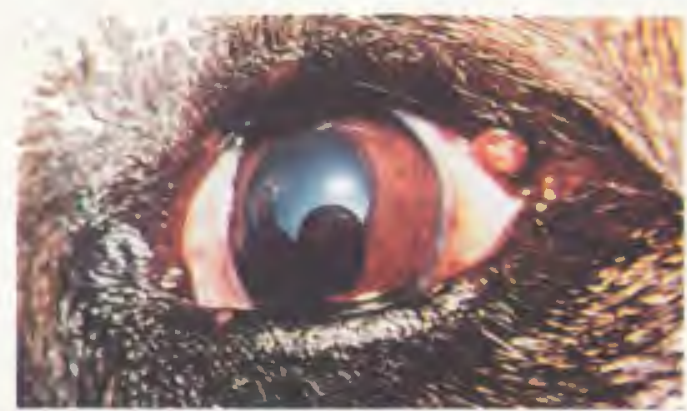

rig 3. Ovejero Alemein, 11 ảjos presentando numerosos quistes uveales

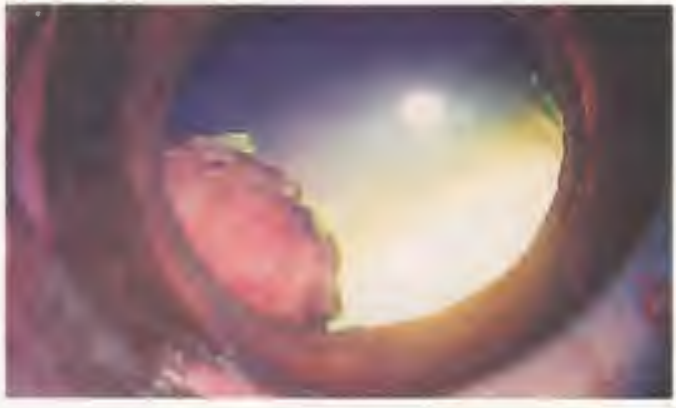

Fig 5. Masa umaral maciza que ex diffenencia con los quistes que san hurooo.

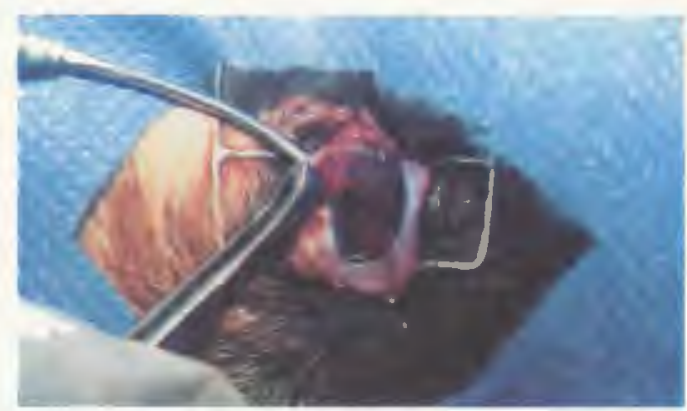

Figura 7. Colocación de la cánula para realizar la aspiración.

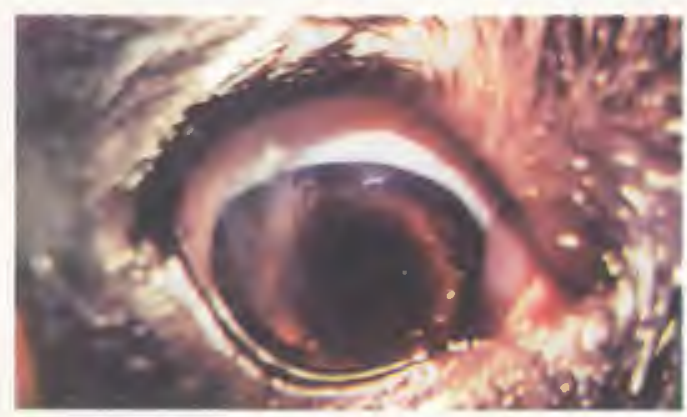

Figura 9. Canino después de los 15 dias de intervenido.

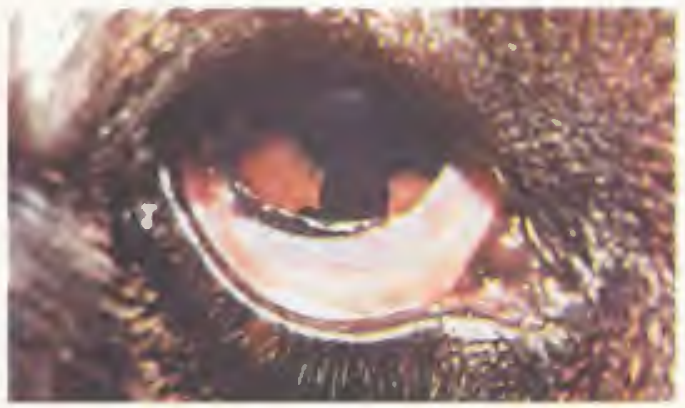

Fig 2. Ovejero Alemán, 8 años presentando 2 quistes uveales.

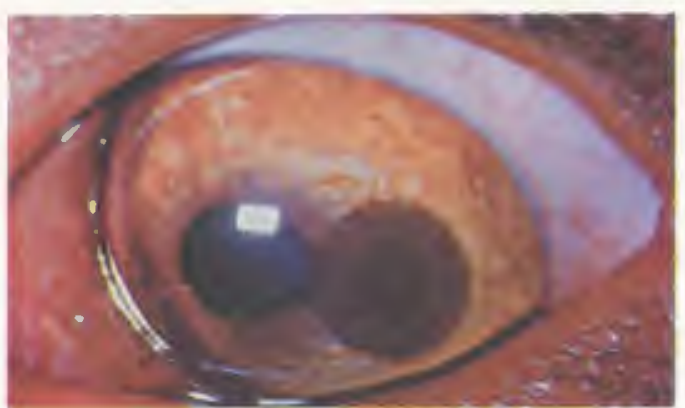

Fig 4. Melanoma iridial para diaznóstico diferencial con quistes uveales.

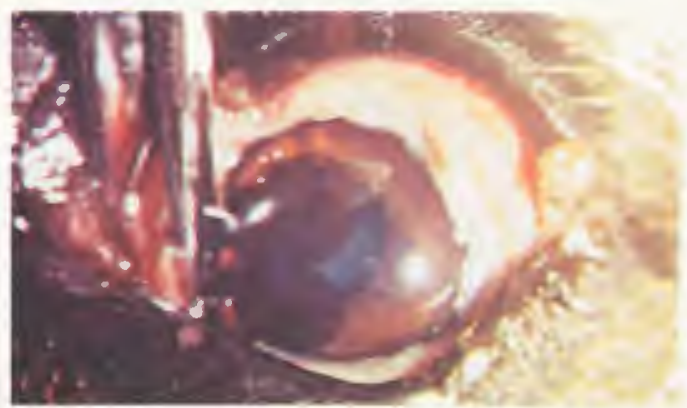

Fig 6. A bondaje cámara anturior en unión limbo exclencomeal.

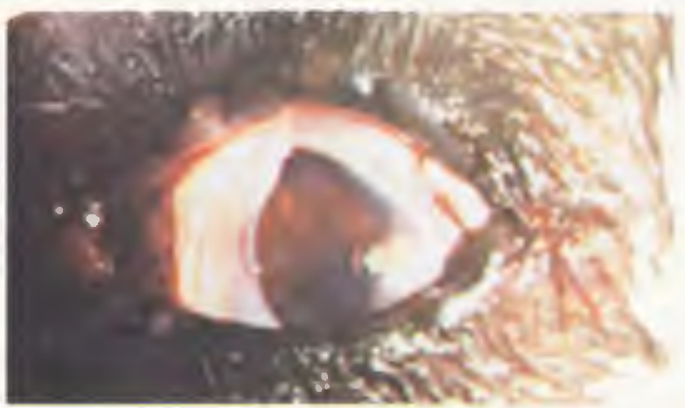

Figura 8. C'anino a las 24 horas de su intervención quirúrgica. 


\section{Literatura Citada}

1. Brooks, D. 1999. Current Concepts in Veter. Ophthalmology. 8:43. USA.

2. Gelatt, K. 1999. Veterinary Ophthalmology. Lippincott Williams \& Wilkins 22:789-792. USA.

3. Gelatt, K.; K. Jonson y R. Peiffer. 1979. Primary iridal pigmented mases in three dogs. J. Am. Anim. Hosp. Assoc. 15:339. USA.

4. Slatter, D. 1992. Fundamentos de Oftalmología Veterinaria. 12:373. Intermédica. USA.

5. Stades, F. 1999. Oftalnologia para el veterinario práctico. 12:145. Intermédica. USA. 